\title{
ON THE BEHAVIOR OF HARMONIC FUNCTIONS NEAR A BOUNDARY POINT
}

\author{
WADE RAMEY AND DAVID ULLRICH
}

\begin{abstract}
Several results on the behavior of harmonic functions at an individual boundary point are obtained. The results apply to positive harmonic functions as well as to Poisson integrals of functions in BMO.
\end{abstract}

I. Introduction. There are several well-known results in the literature concerning the behavior of harmonic functions at an individual boundary point. Perhaps the best-known of these are due to Fatou [F]. Let $\mu$ be a complex Borel measure on $\mathbf{R}$, and let $u$ denote the Poisson integral of $\mu$, so that $u$ is well defined and harmonic in the upper half-plane. Fatou showed that

$$
\lim _{h \rightarrow 0^{+}} \frac{1}{2 h} \int_{-h}^{h} d \mu=L \text { implies } \lim _{y \rightarrow 0^{+}} u(i y)=L,
$$

where $L \in \mathrm{C}$. Fatou also considered nontangential limits: If $F(x)=\int_{-\infty}^{x} d \mu$, then

$$
F^{\prime}(0)=L \text { implies } u \text { has nontangential limit } L \text { at } 0 .
$$

In general the converses of (1) and (2) are false, as Loomis showed in [L]. But in the same paper Loomis proved the converses of (1) and (2) to be valid if the measure $\mu$ is assumed to be positive. Gehring $[\mathbf{G 1}, \mathbf{G 2}]$ obtained many related one-variable results, while Rudin $[\mathbf{R T}]$ and the authors $[\mathbf{R U}]$ considered higherdimensional analogues of the Fatou-Loomis theorems.

One of the goals of the present paper is to show how several of these existing results can be handled by methods which seem to us simpler, or at least more elementary, then the existing proofs. For instance, we prove Rudin's generalization of the Loomis theorem (i.e., if $\mu$ is positive then the converse of (1) holds with $\mathbf{R}^{n}$ in the place of $\mathbf{R}$ ) without recourse to Wiener's tauberian theorem. The main ingredients in our proof (indeed, in all of our proofs) are the basic ideas of normal families and weak $^{*}$-compactness.

But our primary aim in this paper is the application of these techniques in deriving several new results. In Theorem 2.2 , for example, we consider nontangential limits of positive harmonic functions in higher-dimensional half-spaces. (Fatou and Loomis considered only the upper half-plane.) Some rather curious boundary behavior properties of positive harmonic functions are discussed in §III. In addition, these and other results will be shown to hold for Poisson integrals of functions

Received by the editors June 20, 1986.

1980 Mathematics Subject Classification (1985 Revision). Primary 31B25, 31A20; Secondary 30D55, 30D45, 32A40.

The first author is supported by NSF grant DMS-8405382.

(C) 1988 American Mathematical Society $0002-9947 / 88 \$ 1.00+\$ .25$ per page 
in BMO. (See §IV.) Interestingly, some results which fail for positive harmonic functions continue to hold for this latter class of harmonic functions.

We point out what in any event should become clear after reading any of the proofs below: The dilation structure of $\mathbf{R}^{n}$ and $\mathbf{R}_{+}^{n+1}$ plays a central role in all of the results of this paper. The dilations, which of course form a group of self-maps of $\mathbf{R}_{+}^{n+1}$ preserving harmonic functions, open the door for classical "normal families arguments," a luxury one does not find in, say, the open unit ball of $\mathbf{R}^{n+1}$. (The fact that $\mathbf{R}_{+}^{n+1}$ is unbounded creates a few problems of its own, but the trade-off here seems well worthwhile.)

We have benefited from several very helpful conversations with Ralph Howard, especially in connection with the example in 2.9 , and take this opportunity to thank him.

\section{Positive harmonic functions.}

2.1. Preliminaries and notation. The open ball in $\mathbf{R}^{n}$ with center $a$ and radius $r$ will be denoted by $B(a, r)$. If $E \subset \mathbf{R}^{n}, \chi_{E}$ will denote the characteristic function of $E$, and for $r>0, r E$ is the set $\{r x: x \in E\}$. Thus $r B(a, R)=B(r a, r R)$.

Our main setting will be the upper half-space $\mathbf{R}_{+}^{n+1}=\left\{(x, y): x \in \mathbf{R}^{n}, y>0\right\}$, which has $\mathbf{R}^{n}$ as its boundary, and our distinguished boundary point will always be the origin $0 \in \mathbf{R}^{n}$. For $\alpha>0$, we define the cones $\Gamma_{\alpha}=\left\{(x, y) \in \mathbf{R}_{+}^{n+1}:|x|<\alpha y\right\}$. A function $u$ defined in $\mathbf{R}_{+}^{n+1}$ is said to have a nontangential limit $L$ at 0 if, for every $\alpha>0, \lim u(z)=L$ as $z \rightarrow 0$ within $\Gamma_{\alpha}$. If $u$ is bounded in each $\Gamma_{\alpha}$, we will say $u$ is nontangentially bounded at 0 .

The Poisson kernel for $\mathbf{R}_{+}^{n+1}$ is the function

$$
P(x, y)=c_{n} y\left(|x|^{2}+y^{2}\right)^{-(n+1) / 2} \quad\left((x, y) \in \mathbf{R}_{+}^{n+1}\right),
$$

where $c_{n}$ is chosen so that $\int P(x, y) d m(x)=1$; here $d m$ denotes ordinary Lebesgue measure on $\mathbf{R}^{n}$, which we may also write as $d x, d t$, etc. (An integral in which the domain of integration is unspecified will always mean an integral over all of $\mathbf{R}^{n}$.)

The set of all regular positive Borel measures $\mu$ on $\mathbf{R}^{n}$ for which

$$
P[\mu](x, y)=\int P(x-t, y) d \mu(t)
$$

is finite for one (and hence every) $(x, y) \in \mathbf{R}_{+}^{n+1}$ will be denoted by $M$. As is well known, if $\mu \in M$, then $P[\mu]$ is positive and harmonic in $\mathbf{R}_{+}^{n+1}$. Conversely, if $u$ is positive and harmonic in $\mathbf{R}_{+}^{n+1}$, then

$$
u(x, y)=A y+P[u](x, y)
$$

for a unique $A \in[0, \infty)$ and a unique $\mu \in M$. (See [RT].) We will refer to the $\mu$ in (2) as the boundary measure of $u$.

For $\mu \in M$ and $L \in[0, \infty]$ we write $D_{\mathrm{sym}} \mu=L$ if

$$
\lim _{r \rightarrow 0} \frac{\mu(r B)}{m(r B)}=L
$$

for one (and hence every) ball $B$ centered at 0 . We write $D_{0} \mu=L$ if (3) holds for every ball $B$ containing 0 , and $D \mu=L$ if (3) holds for every ball $B \subset \mathbf{R}^{n}$. 
2.2. THEOREM. Suppose $u$ is positive and harmonic in $\mathbf{R}_{+}^{n+1}$, with boundary measure $\mu$, and $L \in[0, \infty)$. Then the following statements are equivalent:

(i) $u$ has nontangential limit $L$ at 0 .

(ii) There exists an $\alpha>0$ such that $\lim u(z)=L$ as $z \rightarrow 0$ within $\Gamma_{\alpha}$.

(iii) $D \mu=L$.

Again, when $n=1$, Fatou showed (iii) $\Rightarrow$ (i) (for arbitrary complex Borel measures on $\mathbf{R}^{1}$ ), while Loomis proved (i) $\Rightarrow$ (iii). (Note that when $n=1, D \mu=L$ if and only if $F^{\prime}(0)=L$, where $F$ is the function defined in the introduction.) When $n>1$, both implications in (i) $\Leftrightarrow$ (iii) appear to be new.

The following theorem is not new, but can be handled in much the same manner as Theorem 2.2.

2.3. THEOREM. Suppose $u$ is positive and harmonic in $\mathbf{R}_{+}^{n+1}$, with boundary measure $\mu$, and $L \in[0, \infty)$. Then $\lim _{y \rightarrow 0} u(0, y)=L$ if and only if $D_{\mathrm{sym}} \mu=L$.

When $n=1$ the assertion $D_{\mathrm{sym}} \mu=L$ implies $\lim _{y \rightarrow 0} u(0, y)=L$ is due to Fatou (again valid for complex measures). Unlike the case for Theorem 2.2, Fatou's onevariable proof extends easily to higher dimensions. In the other direction the results are due to Loomis [L] $(n=1)$ and Rudin [RT] $(n>1)$.

2.4. Before taking up the proofs of Theorems 2.2 and 2.3 we need a few additional preliminaries. Let $C_{c}$ denote the space of functions continuous on $\mathbf{R}^{n}$ with compact support. If $\mu_{1}, \mu_{2}, \ldots, \mu \in M$, we will say that the $\mu_{j}$ converge to $\mu w e a k^{*}$ if $\int \phi d \mu_{j} \rightarrow \int \phi d \mu$ as $j \rightarrow \infty$, for every $\phi \in C_{c}$. This is a slight abuse of terminology since members of $M$ need not belong to the dual space of $C_{c}$.

A sequence $u_{1}, u_{2}, \ldots$ of functions defined on $\mathbf{R}_{+}^{n+1}$ will be said to converge normally if the $u_{j}$ converge uniformly on compact subsets of $\mathbf{R}_{+}^{n+1}$.

2.5. Proposition. If $\mu_{1}, \mu_{2}, \ldots, \mu \in M$ and $P\left[\mu_{j}\right] \rightarrow P[\mu]$ normally, then $\mu_{j} \rightarrow \mu w e a k^{*}$.

ProOF. Let $\phi \in C_{c}$ and $\varepsilon>0$. Set $Q(t)=1 / P(t, 1)$. Then

$$
Q P[\phi](\cdot, y) \rightarrow Q \phi \text { uniformly on } \mathbf{R}^{n} \text { as } y \rightarrow 0
$$

verifying (4) is straightforward given that as $y \rightarrow 0, P[\phi](\cdot, y) \rightarrow \phi$ uniformly on $\mathbf{R}^{n}$ and $Q P[\phi](\cdot, y) \rightarrow 0$ uniformly on $\left\{x \in \mathbf{R}^{n}:|x|>R\right\}$ if $R$ is large enough.

Since $\sup _{j} P\left[\mu_{j}\right](0,1)=\sup _{j} \int P(t, 1) d \mu_{j}<\infty$, we may by (4) fix a $y>0$ so that $\int|\phi-P[\phi](\cdot, y)| d \mu<\varepsilon$ and $\int|\phi-P[\phi](\cdot, y)| d \mu_{j}<\varepsilon$ for every $j$. By Fubini's theorem,

$$
\int P[\phi](\cdot, y) d \mu_{j}=\int \phi P\left[\mu_{j}\right](\cdot, y) d m
$$

the same holding for $\mu$ and $P[\mu]$. Hence

$$
\begin{aligned}
\int \phi d \mu-\int \phi d \mu_{j}= & \int(\phi-P[\phi](\cdot, y)) d \mu \\
& +\int \phi\left(P[\mu](\cdot, y)-P\left[\mu_{j}\right](\cdot, y)\right) d m \\
& +\int(P[\phi](\cdot, y)-\phi) d \mu_{j}=\mathrm{I}+\mathrm{II}+\mathrm{III} .
\end{aligned}
$$

We have seen that $|\mathrm{I}|<\varepsilon$ and $|\mathrm{III}|<\varepsilon$. Since $\phi$ has compact support and $P\left[\mu_{j}\right] \rightarrow$ $P[\mu]$ normally, $|\mathrm{II}|<\varepsilon$ for large $j$. 
2.6. Proposition. Suppose $\mu_{1}, \mu_{2}, \ldots, \mu \in M$ and $\mu_{j} \rightarrow \mu$ weak $k^{*}$. Let $L \in$ $[0, \infty)$. Then $\mu=L m$ if and only if $\mu_{j}(B) \rightarrow L m(B)$ for every open ball $B \subset \mathbf{R}^{n}$.

Proof. Suppose $\mu=L m$. Fix a ball $B \subset \mathbf{R}^{n}$ and let $\varepsilon>0$. Choose $\phi \in C_{c}, \phi \geq$ 0 , such that $\phi=1$ on $B$ and $\int \phi d m<m(B)+\varepsilon$. Then $\varlimsup \mu_{j}(B) \leq \lim \int \phi d \mu_{j}=$ $\int \phi d \mu<L(m(B)+\varepsilon)$. hence $\varlimsup \mu_{j}(B) \leq L m(B)$. Similarly $\underline{\lim } \mu_{j}(B) \geq L m(B)$.

Conversely, suppose $\mu_{j}(B) \rightarrow \operatorname{Lm}(B)$ for every open ball $B \subset \mathbf{R}^{n}$. Fix such a $B=B(a, r)$, let $\varepsilon>0$, and put $B^{\prime}=B(a, r+\varepsilon)$. Choose $\phi \in C_{c}, 0 \leq \phi \leq 1$, such that $\phi=1$ on $B$ and $\phi=0$ off of $B^{\prime}$. Then $\mu(B) \leq \int \phi d \mu=\lim \int \phi d \mu_{j} \leq$ $\lim \mu_{j}\left(B^{\prime}\right)=L m\left(B^{\prime}\right)$. Hence $\mu(B) \leq L m(B)$. Similarly, $\mu(B) \geq L m(B)$. Thus $\mu(B)=L m(B)$ for every open ball $B \subset \mathbf{R}^{n}$, and by regularity, $\mu=L m$.

Although the proof of Proposition 2.6 is quite simple, note that the assumption $\mu_{j} \rightarrow \mu$ weak $^{*}$ need not imply $\mu_{j}(B) \rightarrow \mu(B)$ for every ball $B$ if, say, $\mu$ is a point mass, or even if $\mu=L m$ and this time the $\mu_{j}$ are allowed to be signed measures.

2.7. We also need the following fact, which is very well known: If $\mu \in M$, then the following statements are equivalent; see [Ga, p. 22].

(i) $\sup _{r>0} \mu(B(0, r)) / m(B(0, r))<\infty$.

(ii) $\sup _{y>0} P[\mu](0, y)<\infty$,

(iii) $P[\mu]$ is nontangentially bounded at 0 .

2.8. PROOF OF THEOREM 2.2. Throughout the proof of Theorem 2.2, we assume without loss of generality that the constant $A$ in (2) is 0 , so that $u=P[\mu]$, and that $\mu\left(\mathbf{R}^{n}\right)<\infty$. This last assumption easily implies $\sup _{y>1} P[\mu](0, y)<\infty$.

Obviously (i) implies (ii). Suppose, to reach a contradiction, that (ii) holds and (iii) fails. Then there exists a ball $B_{0} \subset \mathbf{R}^{n}$ and a sequence $r_{j} \rightarrow 0$ such that $\mu\left(r_{j} B_{0}\right) / m\left(r_{j} B_{0}\right)$ fails to converge to $L$ as $j \rightarrow \infty$. Choose a ball $B_{1}$ centered at 0 such that $B_{0} \subset B_{1}$. Then

$$
\frac{\mu\left(r_{j} B_{0}\right)}{m\left(r_{j} B_{0}\right)} \leq \frac{m\left(B_{1}\right)}{m\left(B_{0}\right)} \cdot \frac{\mu\left(r_{j} B_{1}\right)}{m\left(r_{j} B_{1}\right)},
$$

and the latter sequence is bounded by (i) of 2.7 , since (ii) of 2.7 holds. Thus passing to a subsequence, we may assume $\mu\left(r_{j} B_{0}\right) / m\left(r_{j} B_{0}\right) \rightarrow L^{\prime} \neq L, L^{\prime} \in[0, \infty)$.

Put $u_{j}(z)=u\left(r_{j} z\right)$ for $z \in \mathbf{R}_{+}^{n+1}$. Since $u$ is nontangentially bounded at 0 ((iii) of 2.7), the family $\left\{u_{j}\right\}$ is uniformly bounded on compacta, so by passing to a further subsequence we may assume the $u_{j}$ converge normally to a function $v$ harmonic on $\mathbf{R}_{+}^{n+1}$. Since $u$ has limit $L$ within some $\Gamma_{\alpha}, v \equiv L$ in this same $\Gamma_{\alpha}$, which implies $v \equiv L$ on $\mathbf{R}_{+}^{n+1}$.

Now let $\mu_{j}$ be the measure on $\mathbf{R}^{n}$ defined on Borel sets $E$ by the equation $\mu_{j}(E)=r_{j}^{-n} \mu\left(r_{j} E\right)$. Then $u_{j}=P\left[\mu_{j}\right]$, so that $P\left[\mu_{j}\right] \rightarrow P[L m]$ normally. By Propositions 2.5 and 2.6, $\mu_{j}\left(B_{0}\right) \rightarrow \operatorname{Lm}\left(B_{0}\right)$. But $\mu_{j}\left(B_{0}\right)=r_{j}^{-n} \mu\left(r_{j} B_{0}\right) \rightarrow$ $L^{\prime} m\left(B_{0}\right)$, a contradiction.

The proof of (iii) $\Rightarrow$ (i) is much the same in spirit. If (iii) holds and (i) fails, then there exists an $\alpha>0$ and a sequence $\left\{z_{j}\right\} \subset \Gamma_{\alpha}$ such that $z_{j} \rightarrow 0$ while $u\left(z_{j}\right)$ fails to converge to $L$. Since by (iii) of $2.7 u$ is bounded in $\Gamma_{\alpha}$, we may assume $u\left(z_{j}\right) \rightarrow L^{\prime} \neq L, L^{\prime} \in[0, \infty)$. Write $z_{j}=r_{j} z_{j}^{\prime}$, where $z_{j}^{\prime} \in B(0, \alpha) \times\{1\}=K$, a compact subset of $\mathbf{R}_{+}^{n+1}$.

Again set $u_{j}(z)=u\left(r_{j} z\right)$. Then $u_{j}=P\left[\mu_{j}\right]$, where $\mu_{j}$ is defined as before, and the nontangential boundedness of $u$ implies the uniform boundedness of the $u_{j}$ on 
compacta. Passing to a subsequence, we may assume the $u_{j}$ converge normally to a positive harmonic $v$. Write $v=A y+P[\nu]$ as in (2). Since $u(0, y)$ is bounded for $y>0$, the same is true for $v$, hence $A=0$. Thus $P\left[\mu_{j}\right] \rightarrow P[\nu]$ normally, which by Proposition 2.5 implies $\mu_{j} \rightarrow \nu$ weak $^{*}$. But $\mu_{j}(B)=r_{j}^{-n} \mu\left(r_{j} B\right) \rightarrow L m(B)$ for any open ball $B$, since $D \mu=L$. By Proposition 2.6, $\nu=L m$, so that $v=P[\nu] \equiv L$. But then $u_{j} \rightarrow L$ uniformly on $K$, which implies $u_{j}\left(z_{j}^{\prime}\right)=u\left(z_{j}\right) \rightarrow L$, a contradiction.

2.9. AN EXAMPLE. In view of the equivalence of (i) and (ii) of Theorem 2.2 , the reader may be wondering whether (iii) of Theorem 2.2 is equivalent to the condition $D_{0} \mu=L$. The following example shows that it is not.

On $\mathbf{R}^{2}=\mathbf{C}$, define $f\left(r e^{i \theta}\right)=e^{4 i \theta}$. We claim

$$
\int_{B} f d m=0 \text { for every ball } B \text { containing } 0 \text {. }
$$

Supposing (5) is true, define $\mu \in M$ by the equation $d \mu=(1+\cos 4 \theta) d m$. Clearly, $D_{0} \mu=1$. On the other hand, $D \mu$ fails to exist. To see this, choose a ball $B$ such that $\mu(B) / m(B)<1$, and observe $\mu(r B) / m(r B)=\mu(B) / m(B)$ for all $r>0$.

To prove (5) we need only consider balls $B(a, 1)$ with $0<a<1$, since $f\left(r e^{i \theta} z\right)=$ $e^{4 i \theta} f(z)$. The circle with radius 1 and center $a$ has the polar equation $r(\theta)=$ $a \cos \theta+\left(1-a^{2} \sin ^{2} \theta\right)^{1 / 2}$. Hence

$$
\begin{aligned}
\int_{B(a, 1)} f d m & =\int_{0}^{2 \pi} \int_{0}^{r(\theta)} e^{4 i \theta} s d s d \theta \\
& =\frac{1}{2} \int_{0}^{2 \pi} e^{4 i \theta}\left(a^{2} \cos ^{2} \theta+2 a \cos \theta\left(1-a^{2} \sin ^{2} \theta\right)^{1 / 2}+1-a^{2} \sin ^{2} \theta\right) d \theta \\
& =a \int_{0}^{2 \pi} e^{4 i \theta} \cos \theta\left(1-a^{2} \sin ^{2} \theta\right)^{1 / 2} d \theta .
\end{aligned}
$$

If $\theta$ is replaced by $\theta+\pi$, the last integral above is unchanged, but at the same time is transformed into its negative. The integral therefore equals zero.

2.10. A stronger differentiability condition. If $\mu \in M$, the condition $D \mu=L$ implies a seemingly stronger differentiabillity property; this is Proposition 2.10 below. Let us say that a sequence of balls $\left\{B\left(a_{j}, r_{j}\right)\right\}$ is regular $($ at 0$)$ if $r_{j} \rightarrow 0$ and $\left|a_{j}\right| / r_{j}$ is bounded.

2.10. Proposition. Suppose $\mu \in M$ and $D \mu=L$, where $L \in[0, \infty]$. Then $\mu\left(B_{j}\right) / m\left(B_{j}\right) \rightarrow L$ for every regular sequence of balls $\left\{B_{j}\right\}$.

ProOF. Suppose $L<\infty$, the case $L=\infty$ being quite easy. If the proposition is false, there exists a regular sequence $B_{j}=B\left(a_{j}, r_{j}\right)$ such that $\mu\left(B_{j}\right) / m\left(B_{j}\right) \rightarrow$ $L^{\prime} \neq L, L^{\prime} \in[0, \infty) .\left(L^{\prime}<\infty\right.$ since

$$
\mu\left(B_{j}\right) / m\left(B_{j}\right) \leq \mu\left(B\left(0,\left|a_{j}\right|+r_{j}\right)\right) m\left(B\left(0,\left|a_{j}\right|+r_{j}\right)\right)^{-1}\left(\left|a_{j}\right|+r_{j}\right)^{n} r_{j}^{-n} .
$$

On the right-hand side we have symmetric averages multiplied by terms from a bounded sequence, and since $D \mu=L<\infty$, we obtain $\sup _{j} \mu\left(B_{j}\right) / m\left(B_{j}\right)<\infty$.) Passing to a subsequence, we may assume the points $a_{j}^{\prime}=a_{j} / r_{j}$ converge to some $a \in \mathbf{R}^{n}$. The measures $\mu_{j}$, defined as before by $\mu_{j}(E)=r_{j}^{-n} \mu\left(r_{j} E\right)$, converge weak $^{*}$ to $L m$, and a simple variation on the proof of Proposition 2.6 shows then 
that $\mu_{j}\left(B\left(a_{j}^{\prime}, 1\right)\right) \rightarrow \operatorname{Lm}(B(a, 1))$. This is a contradiction, since $\mu_{j}\left(B\left(a_{j}^{\prime}, 1\right)\right)=$ $r_{j}^{-n} \mu\left(B_{j}\right) \rightarrow L^{\prime} m(B(a, 1))$.

2.11. A function $u$ on $\mathbf{R}^{n}$ is radial if $|x|=\left|x^{\prime}\right|$ implies $u(x)=u\left(x^{\prime}\right)$. We will say a function $u(x, y)$ on $\mathbf{R}_{+}^{n+1}$ is radial in $x$ if $u(\cdot, y)$ is radial on $\mathbf{R}^{n}$ for each $y>0$.

In proving Theorem 2.3 we will need the following property of harmonic functions.

2.11. PROPOSITION. Suppose $u(x, y)$ is harmonic on $\mathbf{R}_{+}^{n+1}$, and radial in $x$, and $u(0, y)=0$ for every $y>0$. Then $u \equiv 0$.

PROOF. We may express $u(x, y)$ as a power series near, say, $(0,1)$. Since $u$ is radial in $x$, this power series can be put in the form

$$
u(x, y)=\sum_{k=0}^{\infty} c_{k}(y)|x|^{2 k} .
$$

Applying the Laplacian to both sides of (6) gives

$$
0=\sum_{k=0}^{\infty} c_{k}^{\prime \prime}(y)|x|^{2 k}+\sum_{k=1}^{\infty} c_{k}(y) a_{k}|x|^{2(k-1)},
$$

where $a_{k}=4 k(k-1)+2 k n$. From (7) we obtain the relation $c_{k+1}(y)=c_{k}^{\prime \prime}(y) / a_{k+1}$, $k=0,1, \ldots$ Since $u(0, y)=c_{0}(y) \equiv 0$, we see $c_{k}(y) \equiv 0$ for $k=0,1, \ldots$

2.12. PROOF OF THEOREM 2.3. We may assume $u=P[\mu]$ and $\mu\left(\mathbf{R}^{n}\right)<\infty$. Define a measure $\tilde{\mu}$ on $\mathbf{R}^{n}$, the "radialization of $\mu$," by the equation

$$
\int \phi d \tilde{\mu}=\iint_{S} \phi(|x| \varsigma) d \sigma(\varsigma) d \mu(x) \cdot\left(\phi \in C_{c}\right) .
$$

Here $S$ is the unit sphere on $\mathbf{R}^{n}$ and $\sigma$ is the usual rotation invariant probability measure on $S$. One easily check that $\tilde{\mu}(B(0, r))=\mu(B(0, r))$ for every $r>0$, that $P[\tilde{u}](0, y)=P[\mu](0, y)$ for all $y>0$, and that $P[\tilde{\mu}](x, y)$ is radial in $x$. Now the proof is practically the same as that of Theorem 2.2 .

To show $\lim _{y \rightarrow 0} u(0, y)=L$ implies $D_{\text {sym }} \mu=L$, suppose that $P[\tilde{\mu}](0, y) \rightarrow L$ as $y \rightarrow 0$ but that for some sequence $r_{j} \rightarrow 0, \tilde{\mu}\left(B\left(0, r_{j}\right)\right) / m\left(B\left(0, r_{j}\right)\right) \rightarrow L^{\prime}=L$, $L^{\prime} \in[0, \infty)$. Setting $u_{j}(z)=P[\tilde{\mu}]\left(r_{j} z\right)=P\left[\tilde{\mu}_{j}\right](z)$ as in the proof of Theorem 2.2, we may assume $u_{j} \rightarrow v=P[\nu]$ normally. Clearly $v(0, y)=L$ for all $y>0$, and $v(x, y)$ is radial in $x$. By Proposition $2.11, v \equiv L$ on $\mathbf{R}_{+}^{n+1}$, whence $\nu=$ $L m$. By Propositions 2.5 and 2.6, $\tilde{\mu}_{j}(B(0,1)) \rightarrow L m(B(0,1))$. But $\tilde{\mu}_{j}(B(0,1))=$ $r_{j}^{-n} \tilde{\mu}\left(B\left(0, r_{j}\right)\right) \rightarrow L^{\prime} m(B(0,1))$, a contradiction.

We do not prove the converse, since an easily adapted argument of Fatou proves this for all complex Borel measures. (See [D, p. 4], for the case of the unit circle.)

2.13. The case $L=\infty$. When $L=\infty$, Theorems 2.2 and 2.3 fail; the example in [RT] shows this. The implications $D_{\text {sym }} \mu=\infty \Rightarrow D \mu=\infty \Rightarrow P[\mu] \rightarrow \infty$ nontangentially are nevertheless easily seen to hold in this case.

Poisson integrals of functions in BMO behave better when $L=\infty$; see part IV.

REMARKS. 1. In view of (2) in the introduction, which is valid for all complex Borel measures $\mu$, it is natural to ask the following: If $\mu$ is a complex Borel measure on $\mathbf{R}^{n}, n>1$, does $D \mu=L$ imply $P[\mu]$ has nontangential limit $L$ at 0 ? We have not been able to settle this question. 
2. The techniques of this paper can be used to prove a result completely analogous to Theorem 2.2 for positive "M-harmonic" functions in the Siegel upper half-space of $\mathbf{C}^{n}$, where "nontangential" is replaced by "admissible," euclidean dilations are replaced by certain nonisotropic "dilations," and euclidean balls on $\mathbf{R}^{n}$ are replaced by the corresponding nonisotropic balls on $H^{n}$, the Heisenberg group. (See [RF, pp. 31-32], for some of the definitions.) Perhaps surprisingly, the natural analogue of Theorem 2.3 fails in this setting. In fact, there exist functions $f \in L^{\infty}\left(H^{n}\right)$ for which " $D_{\text {sym }} f "=0$ while the radial limit of " $P[f]$ " is 1 . See $[\mathbf{R U}]$, where the authors prove this for the unit ball in $\mathbf{C}^{n}$.

III. Sectorial limits. The motivation for the material in this part comes from the following result (Gehring [G2] and Loomis [L]), for which we give a simple normal families argument.

3.1. THEOREM. Suppose $u$ is positive and harmonic in $\mathbf{R}_{+}^{2}, 0<\theta_{1}<\theta_{2}<\pi$, and $\lim _{r \rightarrow 1} u\left(r e^{i \theta_{j}}\right)=L_{j}, j=1,2$, where $L_{j} \in[0, \infty)$. Then

$$
\lim _{r \rightarrow 0} u\left(r e^{i \theta}\right)=\frac{L_{2}-L_{1}}{\theta_{2}-\theta_{1}}\left(\theta-\theta_{1}\right)+L_{1}=L(\theta)
$$

for every $\theta \in(0, \pi)$.

Before taking up the proof of Theorem 3.1, we note the following fact, which will be used repeatedly in §III without further mention: Let $r_{1}>r_{2}>\cdots>0$ with $\lim r_{j}=0$. Suppose $u$ is positive and harmonic in $\mathbf{R}_{+}^{n+1}$ and that $\lim _{r \rightarrow 0} u\left(r z_{0}\right)<$ $\infty$ for some $z_{0} \in \mathbf{R}_{+}^{n+1}$. Put $u_{j}(z)=u\left(r_{j} z\right)\left(z \in \mathbf{R}_{+}^{n+1}\right)$. Then some subsequence of $\left\{u_{j}\right\}$ converges normally to a positive harmonic function which is nontangentially bounded at 0 . The proof of this is a straightforward consequence of Harnack's inequalities and we leave it to the reader.

PROOF OF THEOREM 3.1. Suppose for some $\theta_{0} \in(0, \pi)$ there exists a sequence $r_{j} \rightarrow 0$ such that $u\left(r_{j} e^{i \theta}\right) \rightarrow L^{\prime} \neq L\left(\theta_{0}\right)$. Put $u_{j}(z)=u\left(r_{j} z\right)$. Passing to a subsequence, we may assume $u_{j} \rightarrow v$ normally. Clearly $v\left(r e^{i \theta_{j}}\right)=L_{j}$ for all $r>0$, $j=1,2$. The function $w\left(r e^{i \theta}\right)=v\left(r e^{i \theta}\right)-L(\theta)$ is thus bounded and harmonic in the open cone $C=\left\{r e^{i \theta}: 0<r<\infty, \theta_{1}<\theta<\theta_{2}\right\}$, and has boundary values 0 on $\partial C-\{0\}$. Since $C$ is conformally equivalent to the unit disc, we see that $w \equiv 0$ in $C$, which implies $w \equiv 0$ on $\mathbf{R}_{+}^{2}$. This forces $v\left(r e^{i \theta} \cdot\right)=L\left(\theta_{0}\right), 0<r<\infty$, contradicting the fact that $v\left(e^{i \theta} \cdot\right)=\lim _{j \rightarrow \infty} u\left(r_{j} e^{i \theta} \cdot\right)=L^{\prime}$.

3.2. We wish to pursue some generalizations of Theorem 3.1 to $\mathbf{R}_{+}^{n+1}$. For simplicity, we work in $\mathbf{R}_{+}^{3}$; in 3.10 we indicate how to extend our results here to higher dimensions.

By a cone in $\mathbf{R}_{+}^{3}$ we will mean an open set of the form $T\left(\Gamma_{\alpha}\right)$, where $T$ is an orthogonal transformation of $\mathbf{R}^{3}$ and $\Gamma_{\alpha}$ is the cone defined in 2.1 ; we require that $T\left(\Gamma_{\alpha}\right) \subset \Gamma_{\beta}$ for some $\beta>0$. If $C$ is a cone in $\mathbf{R}_{+}^{3}$, a sector of $\partial C$ is either portion of $\partial C$ bounded by two rays in $\partial C$. (By a ray we mean a set of the form $\{r z: 0<r<\infty\}$, where $z \in \mathbf{R}_{+}^{3}$. If $E \subset \mathbf{R}_{+}^{3}, \partial E$ here and from now on denotes the topological boundary of $E$ relative to $\mathbf{R}_{+}^{3}$.)

Suppose $C$ is a cone in $\mathbf{R}_{+}^{3}$ and $u$ is a function defined in $\mathbf{R}_{+}^{3}$. We will say that $u$ has a sectorial limit $L$ within $\partial C$ if $\lim u(z)=L$ as $z \rightarrow 0$ within a sector of $\partial C$. Evidently, some functions $u$ have several different sectorial limits at 0 . But for positive harmonic functions we have the following theorem. 
3.3. THEOREM. Suppose $u$ is positive and harmonic in $\mathbf{R}_{+}^{3}$, and that $u$ has a sectorial limit $L \in[0, \infty)$ within $\partial C$ for some cone $C$ in $\mathbf{R}_{+}^{3}$. Then $u$ has nontangential limit $L$ at 0 .

ProOF. The case $L=\infty$ is simple and we leave it to the reader.

For the case $L<\infty$ we need the following Phragmen-Lindelöf result: If a harmonic function $u$ in $\mathbf{R}_{+}^{3}$ is bounded in a cone $C$ in $\mathbf{R}_{+}^{3}$, and is 0 on $\partial C$, then $u \equiv 0$. To prove this, assume $|u| \leq 1$ in $C$. Put $v\left(x_{1}, x_{2}, y\right)=y+\left(x_{1}^{2}+x_{2}^{2}+y^{2}\right)^{-1 / 2}$; note that $v$ is positive and harmonic in $\mathbf{R}_{+}^{3}$. Let $a>0$. Then $\overline{\lim }(a v-u) \geq 0$ on $\{0\} \cup \partial(C \cap\{0<y<1 / a\})$, so that $u \leq a v$ in $C \cap\{0<y<1 / a\}$ for any $a>0$. It follows that $u \leq 0$ in $C$. Similarly $u \geq 0$ in $C$, whence $u \equiv 0$.

Now suppose $u$ is positive and harmonic in $\mathbf{R}_{+}^{3}$ and that $u$ has a sectorial limit $L<\infty$ within $\partial C$. If $u$ fails to have nontangential limit $L$ at 0 , there exists a sequence $z_{j} \rightarrow 0$ within some $\Gamma_{\alpha}$ such that $u\left(z_{j}\right) \rightarrow L^{\prime} \neq L$. Write $z_{j}=r_{j} z_{j}^{\prime}$, where $z_{j}^{\prime} \in B(0, \alpha) \times\{1\}$. Setting $u_{j}(z)=u\left(r_{j} z\right)$, and passing to a subsequence, we may assume the $u_{j}$ converge normally to a positive harmonic $v$. This $v=L$ on a sector in $\partial C$, since $u$ has sectorial limit $L$ within $\partial C$. Since $v$ is real analytic and $\partial C$ is a real analytic surface in $\mathbf{R}_{+}^{3}, v \equiv L$ on $\partial C$. The nontangential boundedness of $v$ now shows $v \equiv L$ by the result above. Thus $\lim _{j \rightarrow \infty} u_{j}\left(z_{j}^{\prime}\right)=L$, a contradiction.

3.4. RESUlTS RELATED TO THEOREM 3.3. (a) The proof of Theorem 3.3 easily extends to prove a more general result. Let $\gamma$ be a simple, closed, real analytic curve in, say, the plane $\{y=1\} \subset \mathbf{R}_{+}^{3}$. Define the surface $S(\gamma)=\{r z: 0<r<\infty, z \in$ $\gamma$ \}. THEOREM: If $u$ is positive and harmonic in $\mathbf{R}_{+}^{3}$, and $u$ has limit $L$ within a "sector" of $S(\gamma)$, then $u$ has nontangential limit $L$ at 0 .

(b) As concerns surfaces which are not real analytic, Walter Rudin has observed that the following result (which we state a little imprecisely) follows from an argument slightly different from the proof of Theorem 3.3. Suppose $\gamma \subset\{y=1\}$ is a curve which is not real analytic, and put $S(\gamma)=\{r z: 0<r<\infty, z \in \gamma\}$. THEOREM: If $u$ is positive and harmonic in $\mathbf{R}_{+}^{3}$ and $\lim u(z)=L$ as $z \rightarrow 0$ within $S(\gamma)$, then $u$ has nontangential limit $L$ at 0 . PROOF. Suppose not. Then as in the proof of Theorem 3.3, we arrive at a positive harmonic $v \not \equiv L$ such that $v=L$ on $S(\gamma)$. But $S(\gamma)$ is not contained in the zero variety of any nontrivial real analytic function, by our assumption on $\gamma$. Hence $v \equiv L$, a contradiction.

(c) In Theorem 3.3 (and in (a) above) we need only assume there exists a countable collection $R_{1}, R_{2}, \ldots$ of distinct rays in $\partial C$ such that $\lim u(z)=L$ as $z \rightarrow 0$ along each $R_{j}$. To see this, note that the limit function $v$ in the proof of Theorem 3.3 would not be identically $L$ on each $R_{j}$. The rays $R_{j}$ have an accumulation ray somewhere in $\partial C$, and the real analyticity of $v$ again forces $v \equiv L$ on $\partial C$.

3.5. SECTORIAL LIMITS WITHIN HALF-PLANES. A plane $\Lambda$ in $\mathbf{R}^{3}$ through the origin, $\Lambda \neq \mathbf{R}^{2}$, determines a half-plane $\Pi=\mathbf{R}_{+}^{3} \cap \Lambda$; in the remainder of the paper $\Pi$ will always denote such a half-plane. Two distinct rays in $\Pi$ naturally define a sector of $\Pi$; if $u$ is a function defined on $\mathbf{R}_{+}^{3}$, we say $u$ has a sectorial limit $L$ within $\Pi$ if $\lim u(z)=L$ as $z \rightarrow 0$ within a sector of $\Pi$.

It is natural to consider what happens when the rays of Theorem 3.1 are replaced by half-planes $\Pi_{1}, \Pi_{2}$, in the context of $R_{+}^{3}$. We need to distinguish the cases $\Pi_{1} \cap \Pi_{2} \neq \varnothing$ and $\Pi_{1} \cap \Pi_{2}=\varnothing$. 
3.6. THEOREM. Suppose $u$ is positive and harmonic in $\mathbf{R}_{+}^{3}$, and that $u$ has sectorial limits $L_{2}, L_{2} \in[0, \infty)$ within $\Pi_{1}, \Pi_{2}$, respectively, where $\Pi_{1} \cap \Pi_{2} \neq \varnothing$. Then

(i) $L_{1}=L_{2}$,

(ii) If the angle between $\Pi_{1}$ and $\Pi_{2}$ is an irrational multiple of $\pi$, then $u$ has nontangential limit $L_{1}=L_{2}$ at 0 .

PROOF. (i) Some subsequence of the dilates $u_{j}(z)=u\left(2^{-j} z\right)$ converges normally to a harmonic $v$ which is identically $L_{1}$ in a sector of $\Pi_{1}$, and which is identically $L_{2}$ in a sector of $\Pi_{2}$. By analytic continuation, $v=L_{1}$ on $\Pi_{1}, v=L_{2}$ on $\Pi_{2}$. Since $\Pi_{1} \cap \Pi_{2} \neq \varnothing, L_{1}=L_{2}$.

(ii) Put $L=L_{1}=L_{2}$. If $u$ fails to have nontangential limit $L$ at 0 , then as in the proof of Theorem 3.3, some sequence of dilates of $u$ converges normally to a harmonic $v \not \equiv L$ such that $v=L$ on $\Pi_{1} \cup \Pi_{2}$. Fix a ball $B \subset \mathbf{R}_{+}^{3}$ with center on $\Pi_{1} \cap \Pi_{2}$, and let $\theta$ denote the angle between $\Pi_{1}$ and $\Pi_{2}$. Keeping $\Pi_{1}$ fixed, consider the rotates of $\Pi_{2} \cap B$ through angles $\theta, 2 \theta, 3 \theta, \ldots$, using $\Pi_{1} \cap \Pi_{2}$ as the axis of rotation. By the Schwarz reflection principle, $v=L$ on each of these rotates. Since $\theta$ is an irrational multiple of $\pi$, the union of these rotates is dense in $B$. We conclude $v \equiv L$ in $B$, a contradiction.

3.7. EXAMPLES. We give some examples to show that (ii) of Theorem 3.6 fails if the angle between $\Pi_{1}$ and $\Pi_{2}$ is a rational multiple of $\pi$. Introduce polar coordinates $(r, \theta)$ in the $\left(x_{1}, x_{2}\right)$ plane, define $f\left(r e^{i \theta}\right)=\sin 2 \theta$, and let $u$ be the Poisson integral of $f ; u$ is then bounded and harmonic in $\mathbf{R}_{+}^{3}$. Since $f$ is odd with respect to each of $x_{1}$ and $x_{2}$, and the Poisson kernel is even with respect to $x_{1}$ and $x_{2}$, it is clear that $u$ vanishes on the half-planes $\left\{x_{1}=0\right\} \cap \mathbf{R}_{+}^{3}$ and $\left\{x_{2}=0\right\} \cap \mathbf{R}_{+}^{3}$. Yet $u$ fails to have nontangential limit 0 at the origin. To see this, take a point $z \in \mathbf{R}_{+}^{3}$ such that $u(z) \neq 0$, and note $u(r z)=P[f](r z)=P[f(r t)](z)=P[f(t)](z)=u(z)$ for all $r>0$.

For other rational multiples of $\pi$ we need only look among the functions $u_{k}=$ $P[\sin k \theta], k=3, \ldots$, for similar counterexamples.

3.8. In the upper half of the $\left(x_{1}, y\right)$ plane let $\arg \left(x_{1}, y\right)$ denote the principal value argument, and for $\theta \in(0, \pi)$, let $\Pi(\theta)$ denote the half-plane $\left\{\left(x_{1}, x_{2}, y\right) \in\right.$ $\left.\mathbf{R}_{+}^{3}: \arg \left(x_{1}, y\right)=\theta\right\}$.

Theorem 3.8 below handles the case $\Pi_{1} \cap \Pi_{2}=\varnothing$.

3.8. THEOREM. Suppose $u$ is positive and harmonic in $\mathbf{R}_{+}^{3}$, and that $u$ has sectorial limits $L_{1}, L_{2} \in[0, \infty)$ within $\Pi\left(\theta_{1}\right), \Pi\left(\theta_{2}\right)$ respectively, where $0<\theta_{1}<$ $\theta_{2}<\pi$. Put

$$
L(\theta)=\frac{L_{2}-L_{1}}{\theta_{2}-\theta_{1}}\left(\theta-\theta_{1}\right)+L_{1} .
$$

Then for every $\theta \in(0, \pi)$, $u$ has limit $L(\theta)$ within every sector of $\Pi(\theta)$. In the case $L_{1}=L_{2}, u$ has nontangential limit $L_{1}$ at 0 .

PROOF. We first need another Phragmen-Lindelöf result: If $v$ is harmonic on $\mathbf{R}_{+}^{3}$ and is bounded in the infinite wedge $W=\left\{\theta_{1}<\arg \left(x_{1}, y\right)<\theta_{2}\right\}$, then $v=0$ on $\partial W$ implies $v \equiv 0$. The argument used in the proof of Theorem 3.3 can be used here once we find a positive harmonic $w\left(x_{1}, x_{2}, y\right)$ on $\mathbf{R}_{+}^{3}$ such that $w \rightarrow+\infty$ as $y \rightarrow \infty$, as $\left(x_{1}, y\right) \rightarrow(0,0)$, and as $\left|x_{2}\right| \rightarrow \infty$. For this purpose, note that $\alpha(z)=\log \left|2 /\left(1-z^{2}\right)\right|$ is positive and harmonic in the unit disc and satisfies 
$\lim \alpha(z)=+\infty$ as $z \rightarrow 1$ or $z \rightarrow-1$. After a conformal map we obtain a positive harmonic $\beta$ on $\mathbf{R}_{+}^{2}$ such that $\lim \beta(z)=+\infty$ as $z \rightarrow 0$ or $|z| \rightarrow \infty$. The function $w\left(x_{1}, x_{2}, y\right)=\beta\left(x_{1}, y\right)+\beta\left(x_{2}, y\right)$ thus has the desired properties.

Now the proof of Theorem 3.8 is practically the same as the one given for Theorem 3.1. If the theorem is false, we pass to the limit of an appropriate sequence of dilates of $u$ and obtain a positive harmonic $v$ which is identically $L_{1}, L_{2}$ on $\Pi\left(\theta_{1}\right), \Pi\left(\theta_{2}\right)$, respectively, yet $v$ is not identically $L(\theta)$ on $\Pi(\theta)$ for some $\theta \in(0, \pi)$. In the wedge $W=\left\{\theta_{1}<\arg \left(x_{1}, y\right)<\theta_{2}\right\} v$ is easily seen to be bounded. Put $a\left(x_{1}, x_{2}, y\right)=L\left(\arg \left(x_{1}, y\right)\right)$. Then $a$ is bounded and harmonic on $\mathbf{R}_{+}^{3}$. The function $v-a$ is therefore identically 0 by our result above, a contradiction.

3.9. THE CASE $L=\infty$. All of the results in §III remain valid if any of the sectorial limits is assumed to be $+\infty$. This is true because of the following result, whose straightforward proof we omit: If $u$ is positive and harmonic in $\mathbf{R}_{+}^{n+1}$, and $\lim u(z)=\infty$ as $z \rightarrow 0$ along some ray, then $u$ has nontangential limit $\infty$ at 0 .

3.10. Although we have been working exclusively in $\mathbf{R}_{+}^{3}$ in $\S I I I$, natural analogues of all the results here hold in higher dimensions. For instance, if $C$ is a cone in $\mathbf{R}_{+}^{n+1}$ and $\Omega$ is an open subset of $\partial C \cap\{y=1\}$, we may define the "sector" $S(\Omega)=\{r z: z \in \Omega, 0<r<\infty\}$. Sectorial limits within $\partial C$ may then be defined, and a result completely analogous to Theorem 3.3 holds (with the same proof). The other results in §III can be extended in a similar manner.

IV. Poisson integrals of functions in BMO.

4.1. If $B \subset \mathbf{R}^{n}$ is a ball and $f \in L_{\text {loc }}^{1}\left(\mathbf{R}^{n}\right)$, define

$$
f_{B}=\frac{1}{m(B)} \int_{B} f d m \text {. }
$$

$\mathrm{BMO}=\mathrm{BMO}\left(\mathbf{R}^{n}\right)$ is the space of functions $f \in L_{\text {loc }}^{1}\left(\mathbf{R}^{n}\right)$ for which

$$
\|f\|_{\mathrm{BMO}}=\sup _{B} \frac{1}{m(B)} \int_{B}\left|f-f_{B}\right| d m<\infty .
$$

The space BMO arises in nature as the dual space of $H^{1}\left(\mathbf{R}^{n}\right)$, which is classically defined to be the space $\left\{f \in L^{1}\left(\mathbf{R}^{n}\right): R_{j} f \in L^{1}, j=1, \ldots, n\right\}$; here the $R_{j}$ are the Riesz transforms as defined in [St]. The norm in $H^{1}$ is usually taken to be $\|f\|_{H^{1}}=\|f\|_{L^{1}}+\sum\left\|R_{j} f\right\|_{L^{1}}$.

More precisely, BMO, modulo constant functions, is $\left(H^{1}\right)^{*}$ via the pairing

$$
\langle g, f\rangle=\int g f d m .
$$

In (1), $f \in \mathrm{BMO}$, while for (1) to exist as a Lebesgue integral we must assume $g$ belongs to an appropriate dense subspace of $H^{1}$. (The class of functions described in Proposition 4.7 will do.) The norms $\|f\|_{\mathrm{BMO}}$ and $\|f\|_{(H)}$. are equivalent on $\mathrm{BMO} /$ constants. For more information on these topics we refer to [FS and $\mathbf{C W}$ ].

4.2. If $f \in \mathrm{BMO}$, then $\int|f(t)| P(t, 1) d t<\infty$ (see [Ga, p. 234]), so that

$$
P[f](x, y)=\int P(x-t, y) f(t) d t
$$

is a well-defined harmonic function on $\mathbf{R}_{+}^{n+1}$. 
By analogy with $\S 2.1$, we write $D_{\text {sym }} f=L$ if

$$
\lim _{r \rightarrow 0} \frac{1}{m(r B)} \int_{r B} f d m=L
$$

for every ball $B$ centered at 0 , and write $D f=L$ if (2) holds for every ball $B \subset \mathbf{R}^{n}$. We here allow the values $L= \pm \infty$ if $f$ is real-valued; if $f$ is complex-valued $L$ may be the point at infinity.

We wish to show that all of the results of $\S \S I I$ and III (and more) are true for Poisson integrals of functions in BMO. We carry this out mainly for the results in II; once the machinery for this is in place it will be clear the results in III hold here also.

4.3 THEOREM. If $f \in \mathrm{BMO}$ and $L \in \mathrm{C}$, then the following statements are equivalent:

(i) $P[f]$ has nontangential limit $L$ at 0 .

(ii) $P[f](z) \rightarrow L$ as $z \rightarrow 0$ within some $\Gamma_{\alpha}$.

(iii) $D f=L$.

4.4. THEOREM. If $f \in \mathrm{BMO}$ and $L \in \mathbf{C}$, then $\lim _{y \rightarrow 0} P[f](0, y)=L$ if and only if $D_{\text {sym }} f=L$.

In contrast to the situation in 2.13 , we obtain the following positive result when $L=\infty$.

4.5. THEOREM. Suppose $f \in \mathrm{BMO}$ is real-valued. Then the following statements are equivalent:

(i) $D_{\text {sym }} f=+\infty$.

(ii) $\lim _{y \rightarrow 0} P[f](0, y)=+\infty$.

(iii) $P[f]$ has nontangential limit $+\infty$ at 0 ;

(iv) $D f=+\infty$.

As the proof will show, Theorem 4.5 is also valid if $f$ is complex-valued and $+\infty$ is replaced by the point at infinity.

4.6. We wish to make use of the "atomic" description of $H^{1}$. An atom is a function $a \in L^{\infty}\left(\mathbf{R}^{n}\right)$ such that

(i) $\operatorname{supp}(a) \subset B$ and $\|a\|_{\infty} \leq 1 / m(B)$ for some ball $B \subset \mathbf{R}^{n}$, and

(ii) $\int a d m=0$.

The atomic decomposition of $H^{1}$ is then the following result [FS]: $f \in H^{1}$ if and only if there exist atoms $a_{1}, a_{2}, \ldots$ and complex numbers $\lambda_{1}, \lambda_{2}, \ldots$ with $\sum\left|\lambda_{j}\right|<$ $\infty$, such that $f=\sum \lambda_{j} a_{j}$. In this case $\|f\|_{H^{1}}$ is comparable to inf $\sum\left|\lambda_{j}\right|$, the infimum being taken over all atomic decompositions of $f$.

4.7. Proposition. There exists a constant $C<\infty$ such that if $|g(t)| \leq$ $\left(1+|t|^{2}\right)^{-(n+1) / 2}$ and $\int g d m=0$, then $\|g\|_{H^{1}} \leq C$.

Proof. Put $B_{j}=B\left(0,2^{j}\right), j=1,2, \ldots$, and put $A_{1}=B_{1}, A_{j}=B_{j} \backslash B_{j-1}$, $j=2, \ldots$. Define

$$
c_{j}=\frac{1}{m\left(A_{j}\right)} \int_{B_{j}} g d m,
$$

$j=1,2, \ldots$ Set

$$
g_{1}=\left(g-c_{1}\right) \chi_{A_{1}}, \quad \text { and } \quad g_{j}=c_{j-1} \chi_{A_{j-1}}+\left(g-c_{j}\right) \chi_{A_{j}}
$$


for $j=2, \ldots$. Note that since $\int g d m=0$,

$$
\left|\int_{B_{j}} g d m\right|=\left|\int_{\mathbf{R}^{n} \backslash B_{j}} g d m\right|=O\left(2^{-j}\right),
$$

so that $c_{j}=O\left(2^{-(n+1) j}\right)$. This implies $\left\|g_{j}\right\|_{\infty}=O\left(2^{-(n+1) j}\right)$. Now each $g_{j}$ is supported in $B_{j}, \int_{B_{j}} g_{j} d m=0$, and $m\left(B_{j}\right) \approx 2^{j n}$. Thus if $K<\infty$ is large enough, each $a_{j}=2^{j} g_{j} / K$ is an atom, and

$$
g=\sum_{j=1}^{\infty} K 2^{-j} a_{j}
$$

Since we may choose $K$ to be independent of $g,\|g\|_{H^{1}} \leq C$ for some $C<\infty$ independent of $g$.

If $f$ is a function defined on $\mathbf{R}^{n}$, define $f_{r}(t)=f(r t)\left(t \in \mathbf{R}^{n}\right)$.

4.8. PROPOSITION. If $f \in \mathrm{BMO}$, then the following statements are equivalent:

(i) $P[\mid f \|$ is nontangentially bounded at 0 .

(ii) $P[f]$ is nontangentially bounded at 0 .

(iii) $\sup _{0<y<\infty}|P[f](0, y)|<\infty$.

(iv) $\sup _{0<r<\infty}\left|\int_{B(0, r)} f d m\right| / m(B(0, r))<\infty$.

(v) $\sup _{0<r<\infty} \int_{B(0, r)}|f| d m / m(B(0, r))<\infty$.

ProOF. Obviously (i) $\Rightarrow$ (ii) $\Rightarrow$ (iii). To prove (iii) $\Rightarrow$ (iv), note

$$
\|f\|_{\mathrm{BMO}}=\left\|f_{r}\right\|_{\mathrm{BMO}} \text {. }
$$

(This simple but important fact is at the heart of all the results in $\S I V$.) Let $B=B(0,1)$. By Proposition 4.7, $g(t)=\chi_{B}(t) / m(B)-P(t, 1) \in H^{1}$. Thus by $(3)$,

$$
\begin{gathered}
\sup _{0<r<\infty}\left|P[f](0, r)-\frac{1}{m(r B)} \int_{r B} f d m\right| \\
=\sup _{0<r<\infty}\left|\int f_{r} g d m\right|<\infty .
\end{gathered}
$$

(iv) $\Rightarrow(v)$. This follows from the inequality

$$
\begin{aligned}
\frac{1}{m(B)} \int_{B}|f| d m & \leq \frac{1}{m(B)} \int_{B}\left|f-f_{B}\right| d m+\left|f_{B}\right| \\
& \leq\|f\|_{\text {BMO }}+\left|f_{B}\right|
\end{aligned}
$$

valid for any ball $B \subset \mathbf{R}^{n}$.

(v) $\Rightarrow$ (i). This follows directly from the implication (i) $\Rightarrow$ (iii) of 2.7 .

4.9. Proof of ThEOREM 4.3. First note that if $f_{j} \rightarrow f_{0}$ weak $^{*}$ in BMO, $P\left[f_{j}\right] \rightarrow v$ normally, and $P\left[f_{0}\right](0,1)=v(0,1)$, then $P\left[f_{0}\right] \equiv v$. To see this, fix $(x, y) \in \mathbf{R}_{+}^{n+1}$ and set $g(t)=P(x-t, y)-P(t, 1)$. Since $g \in H^{1}$ (Proposition 4.7), $\int\left(f_{j}-f_{0}\right) g d m \rightarrow 0$ as $j \rightarrow \infty$, which implies $v(x, y)=P\left[f_{0}\right](x, y)$.

Now suppose (ii) of Theorem 4.3 is true and (iii) fails. Since (v) of Proposition 4.8 holds, there then exists a ball $B$ and a sequence $r_{j} \rightarrow 0$ such that

$$
\frac{1}{m\left(r_{j} B\right)} \int_{r_{j} B} f d m \rightarrow L^{\prime} \neq L
$$


for some $L^{\prime} \in \mathbf{C}$. By (ii) of Proposition 4.8 and (3), after passing to a subsequence we may assume $P[f]\left(r_{j} z\right)=P\left[f_{r_{j}}\right](z) \rightarrow v(z)$ normally and $f_{r_{j}} \rightarrow f_{0}$ weak $^{*}$ in BMO. Since $f_{0}$ is determined only up to a constant, we may assume $P\left[f_{0}\right](0,1)=L$. But clearly $v \equiv L$ in some $\Gamma_{\alpha}$, since $P[f](z) \rightarrow L$ as $z \rightarrow 0$ within some $\Gamma_{\alpha}$, whence $v \equiv L$. By the remarks above, $P\left[f_{0}\right] \equiv L$, which implies $f_{0} \equiv L$. Setting $g(t)=P(t, 1)-\chi_{B}(t) / m(B)$, we see $\int\left(L-f_{r_{j}}\right) g d m \rightarrow 0$ as $j \rightarrow \infty$, a contradiction.

The proof that (iii) implies (i) is similar. If (i) fails, there exists a sequence $P\left[f_{r_{j}}\right] \rightarrow v$ normally, where $v \not \equiv L$. Passing to a subsequence, we may assume $f_{r_{j}} \rightarrow f_{0}$ weak $^{*}$ in BMO, and that $P\left[f_{0}\right](0,1)=v(0,1)$, so that $v \equiv P\left[f_{0}\right]$. Let $B \subset \mathbf{R}^{n}$ be a ball, and set $g(t)=\chi_{B}(t) / m(B)-P(t, 1)$. Then $\int\left(f_{r}-f_{0}\right) g d m \rightarrow 0$ as $j \rightarrow \infty$, which implies $\int_{B} f_{0} d m / m(B)=L$. Since this is true for every ball $B$, $f_{0} \equiv L$, a contradiction.

4.10. Proof of ThEOREM 4.4. Define

$$
\tilde{f}(x)=\int_{O(n)} f(U x) d U \quad\left(x \in \mathbf{R}^{n}\right) .
$$

Here $O(n)$ is the orthogonal group on $\mathbf{R}^{n}$ and $d U$ is Haar measure on $O(n)$. Once we show that $f \in$ BMO implies $\tilde{f} \in$ BMO, the proof of Theorem 4.4 proceeds exactly along the lines of the last proof, together with the ideas in the proof of Theorem 2.3. But for any ball $B \subset \mathbf{R}^{n}$,

$$
\begin{aligned}
\frac{1}{m(B)} \int_{B}\left|\tilde{f}-\tilde{f}_{B}\right| d m & =\frac{1}{m(B)} \int_{B}\left|\int_{O(n)}\left(f(U x)-f_{U(B)}\right) d U\right| d m(x) \\
& \leq \int_{O(n)} \frac{1}{m(B)} \int_{B}\left|f(U x)-f_{U(B)}\right| d m(x) d U \\
& \leq\|f\|_{\mathrm{BMO}} .
\end{aligned}
$$

4.11. PROOF OF THEOREM 4.5. (i) $\Rightarrow$ (ii). This follows directly from the proof of (iii) $\Rightarrow$ (iv) of Proposition 4.8 .

(ii) $\Rightarrow$ (iii). For any $\alpha>0$,

$$
\sup _{(x, y) \in \Gamma_{\alpha}}\left\|P\left(\frac{x}{y}-t, 1\right)-P(t, 1)\right\|_{H^{1}}<\infty
$$

this follows readily from Proposition 4.7. Thus

$$
\begin{aligned}
\sup _{(x, y) \in \Gamma_{\alpha}}|P[f](x, y)-P[f](0, y)| \\
\quad=\sup _{(x, y) \in \Gamma_{\alpha}}\left|\int f_{y}(t)\left(P\left(\frac{x}{y}-t, 1\right)-P(t, 1)\right) d t\right|<\infty
\end{aligned}
$$

proving (ii) $\Rightarrow$ (iii).

(iii) $\Rightarrow$ (iv). Fix,$(x, y) \in \mathbf{R}_{+}^{n+1}$, put $B=B(x, y)$, and set $g(t)=P(t, 1)-$ $\chi_{B}(t) / m(B)$. Then

$$
\begin{aligned}
\sup _{0<r<\infty}\left|P[f](r x, r y)-\frac{1}{m(r B)} \int_{r B} f d m\right| \\
=\sup _{0<r<\infty}\left|\int f_{r y}\left(t+\frac{x}{y}\right) g(t) d t\right|<\infty,
\end{aligned}
$$

since $\left\|f_{r y}(t-x / y)\right\|_{\mathrm{BMO}}=\|f\|_{\mathrm{BMO}}$. 
4.12. A Theorem for $\mathrm{BMOA}(\mathbf{R})$. We conclude by pointing out that more can be said if $f \in \operatorname{BMOA}(\mathbf{R})=\left\{f \in \operatorname{BMO}(\mathbf{R}): P[f]\right.$ is holomorphic in $\left.\mathbf{R}_{+}^{2}\right\}$.

4.12. THEOREM. If $f \in \operatorname{BMOA}(\mathbf{R})$ and $L \in \mathbf{C} \cup\{\infty\}$, then the following statements are equivalent:

(i) $\lim _{h \rightarrow 0^{+}}(1 / h) \int_{0}^{h} f(x) d x=L$.

(ii) $\lim _{h \rightarrow 0^{+}}(1 / h) \int_{-h}^{0} f(x) d x=L$.

(iii) $\lim _{h \rightarrow 0^{+}}(1 / 2 h) \int_{-h}^{h} f(x) d x=L$.

(iv) $\lim _{y \rightarrow 0^{+}} P[f](i y)=L$.

(v) $P[f]$ has nontangential limit $L$ at 0 .

This result, for $H^{\infty}(\mathbf{R}) \varsubsetneqq \operatorname{BMOA}(\mathbf{R})$, was obtained by H. S. Shapiro in $[\mathbf{S}]$.

PROOF. We omit the details, except to point out that the theorem follows from our usual dilations - normal families - weak* compactness arguments, together with the following elementary facts: $\operatorname{BMOA}(\mathbf{R})$ is weak ${ }^{*}$-closed in $\operatorname{BMO}(\mathbf{R})$, and if $f \in \operatorname{BMOA}(\mathbf{R})$, then (i) $f=0$ a.e. in $(0, \infty)$ implies $f=0$ a.e. on $\mathbf{R}$, and (ii) $P[f](i y)=0$ for $y \in(0, \infty)$ implies $P[f] \equiv 0$.

REMARK. The results of $\S I I I$ depended only on certain nontangential boundedness properties of positive harmonic functions. Proposition 4.8 shows these same properties hold for $P[f], f \in \operatorname{BMO}\left(\mathbf{R}^{n}\right)$, so that all of the results of $\S$ III are valid for Poisson integrals of BMO functions.

\section{REFERENCES}

[CW] R. R. Coifman and G. Weiss, Extensions of Hardy spaces and their uses in analysis, Bull. Amer. Math. Soc. (N.S.) 83 (1977), 569-646.

[D] P. Duren, Theory of $H^{p}$ spaces, Academic Press, New York, 1970.

[F] P. Fatou, Series trigonometriques et series de Taylor, Acta Math. 30 (1906), 335-400.

[FS] C. Fefferman and E. M. Stein, $H^{p}$ spaces of several variables, Acta Math. 129 (1972), 137-193.

[Ga] J. Garnett, Bounded analytic functions, Academic Press, New York, 1981.

[G1] F. W. Gehring, The Fatou theorem and its converse, Trans. Amer. Math. Soc. 85 (1957), 106-121.

[G2] Harmonic functions and tauberian theorems, Proc. London Math. Soc. (3) 10 (1960), 88-106.

[L] L. H. Loomis, The converse of the Fatou theorem for positive harmonic functions, Trans. Amer. Math. Soc. 53 (1943), 239-250.

[RU] W. Ramey and D. Ullrich, The pointwise Fatou theorem and its converse for positive pluriharmonic functions, Duke Math. J. 49 (1982), 655-675.

[RF] W. Rudin, Function theory in the unit ball of $\mathbf{C}^{n}$, Springer-Verlag, New York, 1980.

[RT] _ Tauberian theorems for positive harmonic functions, Nederl. Akad. Wetensch. Proc. Ser. A 81 (1978), 376-384.

[S] H. S. Shapiro, Boundary values of bounded holomorphic functions of several variabies, Bull. Amer. Math. Soc. (N.S.) 77 (1971), 111-116.

[St] E. M. Stein, Singular integrals and differentiability properties of functions, Princeton Univ. Press, Princeton, N. J., 1970.

Department of Mathematics, Michigan State University, East Lansing, MICHIGAN 48824

Department of Mathematics, Oklahoma State University, Stillwater, OKLAHOMA 74078 